\title{
Influence of social network sites in healthy behavior related to vigorous recreational physical activity
}

DOI: http://doi.org/10.26758/8.1.15

Diego Oswaldo Camacho Vega

Faculty of Medicine and Psychology, Universidad Autónoma de Baja California,

Address correspondence to: Diego Oswaldo Camacho Vega, Email: diego.camacho@uabc.edu.mx

\begin{abstract}
Objectives. Social network sites have become the web applications most used in Mexico facilitated by the use of smartphones and laptops. They have shown influence in the acquisition of healthy behaviors. The objective of this study is to determine if the use of social networking sites helps to improve vigorous recreational physical behavior.

Material and methods. In order to achieve this objective, it was necessary a systematic cluster sampling from 1927 higher education students resulting in 62 subjects who were studying sports in the largest university in Baja California, Mexico in 2017. A questionnaire based on the socialcognitive theory (eHealth Literacy Scales, developed by Norman and Skinner in 2016) was translated into Spanish and adapted to measure the consumer comfort and skill in using social network sites for improving vigorous recreational physical behavior.

Results. An excellent internal reliability of .958 was obtained for the applied test. Regarding the research question about if the use of social networking sites for the improvement of the vigorous recreational physical activity, this study can reject the null hypothesis and conclude that there are statistically significant differences in the response Agree (4) compared to either Strongly Disagree (1), Disagree (2), Undecided (3), and Strongly Agree (5) for items 21, 22, 23, 24, 25, 26, and, 27.

Conclusions. Students who do vigorous exercise are paying attention to the health-related resources available on the SNS and are using them critically to find help related to their recreational activity (sports). Thus, from the perspective of the eHealth Literacy, SNS could be considered as a media that could provide important information to promote healthy behavior regarding the vigorous physical activity.
\end{abstract}

Keywords: social network sites; healthy behavior; physical activity.

\section{Introduction}

Physical inactivity has been identified as the fourth leading risk factor for global mortality causing an estimated 3.2 million deaths globally, according to the World Health Organization (WHO, 2017, p.1). The same source reports that physical activity improves cardiorespiratory and muscular fitness, bone health, reduces the risk of no communicable diseases and their risk factors (NCDs) and depression. Doing regular to moderate physical exercises like sports or fitness bring more benefits for health: "regular moderate intensity physical activity - such as walking, cycling, or participating in sports - has significant benefits for health. For instance, it can reduce the risk of cardiovascular diseases, diabetes, colon and breast cancer, and depression. Moreover, adequate 
levels of physical activity will decrease the risk of a hip or vertebral fracture and help control weight"(WHO, 2107, p.1).

Nonetheless, access to the information through the Social networking sites, with around 2.46 billions of worldwide users in 2017 (***statista, 2017), involves different responsibilities and opportunities to develop healthy behaviors (Norman and Skinner, 2006). Thus, social networking sites (SNS) have become an important media based on Internet, Facebook being the most popular of them, with 1.8 billion monthly active users: "if the social media giant were a country, it would be much bigger than China" (***World Economic Forum, 2017). SNS have become important applications for sharing personal issues, getting improvement and motivation in the realization of physical activity like exercise (Boonwattanopas, 2016).

Thus, the eHealth literacy understood as "the degree to which individuals have the capacity to obtain, process, and understand basic health information and services needed to make appropriate health decisions" (Ratzan and Parker, 2000, p.vi) - in this case, exercise - is a topic that need attention in order to generate health education, "to influence not only individual lifestyle decisions, but also raises awareness of the determinants of health, and encourages individual and collective actions which may lead to a modification of these determinants" (WHO, 2017, p.1).

Looking for a better compression of the social networking sites and applications and eHealth, the main objective of this study is to determine if the use of social media helps to improve vigorous recreational physical behavior.

\section{Theoretical framework}

\section{The physical activity, sport and exercise}

This paper focuses on the study of exercise related to SNS. Nonetheless, the definition of exercise should be differentiated from those of physical activity and sport. First, according to the World Health Organization (WHO, 2017, p.1), physical activity is defined as "any bodily movement produced by skeletal muscles that require energy expenditure". The benefits of physical activity depend on the intensity and time. For adults, WHO recommends the following levels of intensity and duration:

"1. Adults aged 18-64 should do at least 150 minutes of moderate-intensity aerobic physical activity throughout the week or do at least 75 minutes of vigorous-intensity aerobic physical activity throughout the week or an equivalent combination of moderateand vigorous-intensity activity.

2. Aerobic activity should be performed in bouts of at least 10 minutes duration.

3. For additional health benefits, adults should increase their moderate-intensity aerobic physical activity to 300 minutes per week, or engage in 150 minutes of vigorous-intensity aerobic physical activity per week, or an equivalent combination of moderate- and vigorous-intensity activity.

4. Muscle-strengthening activities should be done involving major muscle groups on 2 or more days a week." (WHO, 2017, p.1)

Meanwhile, sport comprises "a range of activities that are highly organized and involve rules, complex skills, and tactics, physical exertion and competition between participants" (O'Keeffe et al., 2016, p.5).

Exercise, for its parts, is a "physical activity that is planned or structured. It involves repetitive body movement done to improve or maintain one or more components of fitness" 
(O'Keeffe et al., 2016, p.5). In short, sports and exercise are included in the physical activity but differentiated by the goal of the activity as well as the level of organization, planning, and structuring. Physical activity is defined as "any activity that involves or requires some form of physical exertion. It includes sport, play, active transport, chores, games, fitness activities, recreation and some forms of work" (O'Keeffe et al., 2016, p.4).

The physical activity has various influences translated into enablers and barriers: social, cultural and environmental. The social enablers and barriers are represented by friends, parents, siblings, neighbors, etc. For its part, the cultural enablers and barriers refer to ethnicity, religion, belief, and values. Last, the environmental enablers and barriers are the climate, location, safety features, etc. this study focused in social influencers, for this reason, they have been defined widely in relation to social networking sites and the human behavior.

Based on the beforehand mentioned considerations, the present study considered the category of recreational physical activity so as to include sports.

\section{eHealth literacy}

In order to understand how the use of technology could facilitate healthy behaviors such as exercise, social cognitive theory looks like adequate theoretical framework due to definition of health literacy: "the cognitive and social skills which determine the motivation and ability of individuals to gain access to, understand and use information in ways which promote and maintain good health" (WHO, 2017).

The foundations of the eHealth literacy concept are based in part on social cognitive theory and self-efficacy theory (Bandura, 1997), which promote competencies and confidence as precursors to behavior change and skill development" (Norman and Skinner, 2006).

Norman and Skinner's eHealth literacy model is comprised of six core skills or literacies: (1) traditional literacy, (2) health literacy, (3) information literacy, (4) scientific literacy, (5) media literacy, and (6) computer literacy (Norman and Skinner, 2006, p.2). For the purposes of this study, the health literacy related to technologies is considered. For instance, the eHealth literacy refers to "the capacity to use and understand the content of interactive health information technologies" (Gilstad, 2014, p.1).

Nonetheless, promoting and maintaining good health through technology requires the development of skills in using technology as a tool.

The Social networking sites era equivalent to health literacy is eHealth literacy, which includes basic literacy as well as information, media, health, computer and scientific literacies (Norman, \& Skinner, 2006). For this reason, youth can serve as an ideal group to test a measure of eHealth literacy given this population's high familiarity with technology (Gilstad, 2014). Thus, the adequate development of eHealth tools could protect consumers from harm and empower them at the same time, because "regardless of the population of interest, the need to navigate the Social networking sites with confidence is particularly important for health issues in which the consequences of using low quality, misleading, or false information are great" (Gilstad, 2014, p.2)

\section{Research question} activity?

Does the use of social networking sites help to improve the vigorous recreational physical 


\section{Hypothesis} activity.

The use of social networking sites helps to improve the vigorous recreational physical

\section{Material and Methods}

A transactional descriptive study involving one sample selected from a statistical population was conducted.

The systematic sampling was conducted considering a sizeable population of 1927 students, with a confidence level of 90 percent and a margin of error of 10\%. 62 students enrolled in the Faculty of Sports in the largest university in Baja California, Mexico, participated voluntarily in the study, male $(\mathrm{N}=50)$ and female $(\mathrm{N}=12)$. Originally, there were 67 students in the sample, but five students were excluded because they did not fulfill the main characteristic of the study population, which was to perform vigorous physical activity.

To achieve the purposes of the study, an adaptation and Spanish translation of the eHEALS scale (eHealth Literacy Scales) developed by Norman and Skinner (2016) was realized. The adaptation consisted of changing all general items that ask for the use of Internet in a general sense to address specifically the use of social networking sites (all the items). From the Global Physical Activity Questionnaire (GPAQ), elaborated by the World Health Organization (2010), five questions were selected for assessing the level of intensity (moderate or vigorous) and duration (hours per day, days per week) of the reported physical recreational activity.

The statistical procedure for the data set has been as follows: 1) the internal consistency of the scale was determined; 2) considering $\mathrm{p}>.05$ level of significance, the Kolmogorov-Smirnov $\mathrm{Z}$ test to assess the normality of variances was calculated; 3) according to the result of the normality test, it was not necessary to calculate a homogeneity test; 4) Non parametric analysis for all items was calculated, according to the results of the Kolmogorov-Smirnov $\mathrm{Z}$ test.

\section{Results}

The internal consistency reliability of the overall scale, as measured by the Cronbach Alfa coefficient, was very high $(\alpha=.958)$.

Regarding the results of the Kolmogorov-Smirnov $\mathrm{Z}$ test, this revealed not normally distributed data for all the items of the instrument; therefore, a nonparametric analysis of the dataset was necessary, considering $\mathrm{p}>.05$ level of significance. For this reason, the Chi-Square nonparametric test, mean, standard deviation, and percentages were calculated for the scale.

The Chi-Square results show significance for items Nr. 21 (MD=3.3387; Std. Deviation=1.21390; p < 0.001), Nr. 22 (MD=3.3387; Std. Deviation=1.21390; p < 0.001), Nr. 23 $(\mathrm{MD}=3.3387$; Std. Deviation=1.21390; p < 0.000), Nr. $24(\mathrm{MD}=3.3387$; Std. Deviation=1.21390; $<0.000)$, Nr. 25 (MD=3.3387; Std. Deviation=1.21390; p < 0.000), Nr. 26 (MD=3.3387; Std. Deviation=1.21390; $\mathrm{p}<0.000)$, Nr. 27 (MD=3.3387; Std. Deviation=1.21390; $\mathrm{p}<0.000)$.

Regarding the research question, if the use of social networking sites helps or not to improve the vigorous recreational physical activity, this study can reject the null hypothesis - based on the items mentioned beforehand - and conclude that there are statistically significant differences in the response Undecided (3) for most items. 
Table 1. Mean, Standard Deviation (std), Chi-Square, and frequencies overall the scale.

\begin{tabular}{lllllllll}
\hline Item & 21 & 22 & 23 & 24 & 25 & 26 & 27 & 28 \\
\hline Mean & 3.3387 & 3.4839 & 3.5161 & 3.3710 & 3.4355 & 3.3710 & 3.4194 & 3.1613 \\
Std. & 1.21390 & 1.31501 & 1.25112 & 1.19059 & 1.13976 & 1.07481 & 1.20855 & 1.23067 \\
Deviation & & & & & & & & \\
Chi- & $19.774^{\mathrm{a}}$ & $18.484^{\mathrm{a}}$ & $23.806^{\mathrm{a}}$ & $25.903^{\mathrm{a}}$ & $39.452^{\mathrm{a}}$ & $33.000^{\mathrm{a}}$ & $27.839^{\mathrm{a}}$ & $9.290^{\mathrm{a}}$ \\
Square & & & & & & & & \\
Asymp. & 0.001 & 0.001 & 0.000 & 0.000 & 0.000 & 0.000 & 0.000 & 0.054 \\
Sig. & & & & & & & & \\
Scale & Frequencies & & & & & & \\
$\mathbf{1}$ & 9.7 & 12.9 & 9.7 & 11.3 & 9.7 & 9.7 & 12.9 & 12.9 \\
$\mathbf{2}$ & 17.7 & 11.3 & 14.5 & 11.3 & 11.3 & 6.5 & 6.5 & 16.1 \\
$\mathbf{3}$ & 16.1 & 12.9 & 11.3 & 19.4 & 16.1 & 30.6 & 21.0 & 25.8 \\
$\mathbf{4}$ & 41.9 & 40.3 & 43.5 & 45.2 & 51.6 & 43.5 & 45.2 & 32.3 \\
$\mathbf{5}$ & 14.5 & 22.6 & 21.0 & 12.9 & 11.3 & 9.7 & 14.5 & 12.9 \\
\hline
\end{tabular}

\section{Discussion and Conclusions}

This research has found that social networking sites have not a clear influence on Health behaviors, supporting partially the suggestion made by Latking \& Knowlton (2015) that social media has been successfully used for online self-help to promote health behaviors, mainly for the ease of accessibility for many individuals.

More information is necessary to affirm a positive influence of SNS on health behaviors. Our results showed that higher percentages were obtained for the undecided options, indicating that students are not sure with regards to a series of issues: about what health resources are available on the social networking sites (item 21), where to find helpful health resources on the social networking sites (item 22), how to find helpful health resources on the social networking sites (item 23), how to use the social networking sites to answer their questions about health (item 24), how to use the health information that they find on the social networking sites to help themselves (item 25). At the same time, they hesitate to assert the confidence about the skills they need to evaluate the health resources they find on the social networking sites (item 26), and the capacity to discriminate highquality from low-quality health resources on the social networking sites (item 27).

Thus, persons who do vigorous exercise are paying attention to the health-related resources available on the SNS and are using them critically to find help related to their recreational activity (including sports), but it is not clear if they are getting basic health information (health literacy) from SNS. 
Other topics that should be evaluated are whether social networking sites provide a significantly greater source of social incentives for increasing physical activity in addition to social support (Zhang et al., 2016) and the critical use of information provided by SNS about health.

Finally, it is important to note that the main social network site mentioned by the students has been Facebook (32\%), followed by Twitter (24\%), which indicates the necessity of considering the characteristics of the sample, the context and the own characteristics of the social networking site in future research.

\section{Research limitations and future research directions}

For a better understanding of the impact of SNS on eHealth Literacy, it is necessary to conduct supplementary research, based on empirical data, considering experimental conditions and increasing the sample size, and to compare low, moderate, and vigorous recreational physical activity. Additionally, the context and specific characteristics of the recreational activities should be analyzed.

\section{References:}

1. Bandura, A., 1997. Self-efficacy: the exercise of control. New York: W.H. Freeman.

2. Boonwattanopas, N., (n.d.). Use of Online Social Media and eHealth Literacy of Urban Youth in Phuket Province, Thailand, 48-62. Available at: https://www.tcithaijo.org/index.php/EAUHJSci/article/download/52898/45632 [Accessed 18 January 2018].]

3. Boyd, N., and Ellison, N., 2008. Social Network Sites: Definition, History, and Scholarship. Journal of Computer-Mediated Communication, 13, pp.210-230.

4. Gilstad, H., 2014. Toward a comprehensive model of eHealth literacy. In: 2nd European Workshop on Practical Aspects of Health Informatics (Pahi). Trondheim, Norway, 19-20 May. CEUR Workshop Proceedings, 1251(Pahi), pp.63-72. https://doi.org/10.13140/2.1.4569.0247

5. Latkin, C. A., and Knowlton, A. R., 2015. Social Network Assessments and Interventions for Health Behavior Change: A Critical Review. Behavioral Medicine, 41(3), pp.90-97. https://doi.org/10.1080/08964289.2015.1034645.

6. Norman, C. D., and Skinner, H. A., 2006. eHealth Literacy: Essential Skills for Consumer Health in a Networked World. Journal of Medical Social networking sites Research, 8(2), e9. http://doi.org/10.2196/jmir.8.2.e9

7. O'Keeffe, M., Walsh, K., Cleary, V., Millar, S., \& Quinlan, M., 2016. Live it Up 1 VCE Units $1 \& 24 e$ eBookPLUS \& Print + studyON. Richmond, Vic. John Wiley \& Sons Australia, Ltd. Available at: https://www.wiley.com/en-

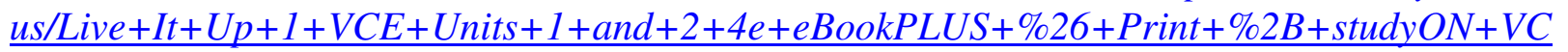
E + Physical + Education + Units $+1+$ and $+2+2 e-p-9780730329374$ [Accesed 24 January 2018]

8. Ratzan, S.C., and Parker, R.M., 2000. Introduction. In: C.R. Selden, M. Zorn, S. Ratzan, R.M. Parker, eds. National Library of Medicine Current Bibliographies in Medicine: Health Literacy. Bethesda, MD: National Institutes of Health.

9. World Economic Forum, 2017, The world's most popular social networks, mapped. [online] Available at: https://www.weforum.org/agenda/2017/03/most-popular-social-networks-mapped/ [Accessed 27 July 2017].

10. World Health Organization, 2017. Health topics. Physical activity [online] Available at: http://www.who.int/topics/physical_activity/en/ [Accessed 24 July 2017].

11. World Health Organization, 2017, Global Strategy on Diet, Physical Activity and Health: 
Physical Activity and adults. [online] Available at: http://www.who.int/dietphysicalactivity/factsheet_adults/en/ [Accessed 24 July 2017].

12. World Health Organization, 2017. Health Promotion: Health literacy and health behavior. [online] Available at: http://www.who.int/healthpromotion/conferences/7gchp/track2/en/ [Accessed 26 July 2017].

13. World Health Organization, 2010. Global Physical Activity Questionnaire. [online] Available at: http://www.who.int/ncds/surveillance/steps/GPAQ/en/ [Accessed 24 July 2017].

14. Zhang, J., Brackbill, D., Yang, S., Becker, J., Herbert, N., and Centola, D., 2016. Support or competition? How online social networks increase physical activity: A randomized controlled trial. Preventive Medicine Reports, 4, pp.453-458. https://doi.org/10.1016/j.pmedr.2016.08.008

15. ***statista, 2017 Number of social network users worldwide from 2010 to 2021 (in billions). Available at: https://www.statista.com/statistics/278414/number-of-worldwide-social-networkusers/ [Accesed 27 March 2018] 